\title{
New approach to VFR pilot training
}

\section{Kristýna Vodičková ${ }^{1 *}$}

\author{
${ }^{1}$ Department of Air Transport, Faculty of Transportation Sciences, Czech Technical University in Prague, Prague, \\ Czech Republic \\ *Corresponding author: Czech Technical University in Prague, Faculty of Transportation Sciences, Department of Air \\ Transport, Horská 3, 12803 Prague, Czech Republic, Email: vodickri@fd.cvut.cz
}

\begin{abstract}
With an increased usage of GNSS navigation devices as one of the primary navigation means during VFR flights, it was necessary to address the issue of proper usage of such devices and to educate pilots about this new trend. Until a recent period, there was no existent comprehensive material that would enable pilots in training to get access to new evolution in navigation. Therefore, a project named CaBilAvi (Capacity Building for Aviation Stakeholders) was launched under the HORIZON 2020 framework. In this article, the author addresses the changes that were derived in VFR pilot training syllabus and pilot education materials. Furthermore, the author mentions specific activities introduced during the project, that were necessary to support the dissemination of new knowledge to the proper audience interested in general aviation.
\end{abstract}

Keywords

GNSS; VFR training; Learning material; New VFR training syllabus; Aviation safety; General aviation

\section{Introduction}

The use of GNSS (Global Satellite Navigation System) applications has in the past decade become widely popular as a primary source of navigation within VFR (Visual Flight Rules) and IFR (Instrument Flight Rules) pilots. Until a recent period, there was not available sufficient knowledge about the proper use of GNSS, that would be comprehensively processed. Therefore, with the launch of the CaBilAvi project, such issue as well as other relevant issues regarding pilot education have been addressed.

Many areas of industry and transportation are in the current decade dependent of the use of Global Navigation Satellite System (GNSS). GNSS system enables high accuracy positioning. With an increasing use of this system, we are encountering large economic impact as well as increased safety of all means of transportation as well as all industry sectors.
The market with GNSS devices and applications keeps growing and this is highly connected to aviation.[1]

With the launch of EGNOS (European Geostationary Navigation Overlay Service), a system providing differential correction signal for GPS (Global Positioning System) over Europe increasing precision to positioning. The EGNOS application is being developed by European Space Agency (ESA), European Commission (EC) and European Organization for the Safety of Air Navigation (EUROCONTROL). EGNOS provides three key services - Open Service, EGNOS Data Access Service and Safety of Life Service (available for safety critical applications, especially in civil aviation, where this service provides enhanced performance, including integrity warning system).[2]

Using EGNOS opens regional airports to general aviation (GA), which is due to limited slot capacity and due to high 
cost of flying into commercial airports quite dependent on these airports (applicable to IFR general aviation).[3]

The use of GNSS is convenient and becoming widely popular within pilots of general aviation. This has entailed a growing issue of pilot education about the proper use of GNSS devices for navigation. As a support to the growing use of GNSS, a coordination and support type project CaBilAvi (Capacity Building for Aviation stakeholders) targeting the spreading of awareness and knowledge about European navigation satellite systems Galileo and EGNOS has been launched under the HORIZON2020 framework with a direct support of the GSA (European Global Navigation Satellite System Agency). The project is targeting the above-mentioned issues - EGNOS adoption in aviation and updating the education and training materials for GA pilots including implementation of section regarding GNSS.

The following text is an overview of an approach to the project, work done and the outcomes, the project has brought. Further, this text continues with information regarding the integration of new knowledge about GNSS into pilot training courses (applicable for VFR), with description of activities introduced, secondly the text focuses on information regarding dissemination of information about GNSS within amateur and professional aviation community.

\section{Objective of CaBilAvi}

The CaBilAvi project consisted of four specific objectives (SO) (SO3 and SO4 discussed in this article), together aiming to meet two high level project objectives (HLO). Work carried out during the project was done in parallel, to meet all the objectives at the end of the project and was integrated into work packages related to each specific objective. The SO3 was focused on updating the requirements and guidelines to develop new curricula for pilot training with included information about RNP operations with an effect of an increased awareness about EGNSS in aviation throughout Europe. The goal of SO4 was to disseminate the aforementioned information to increase awareness about EGNSS within the European amateur and professional aviation community.

Further the article focuses on the SO3 and SO4 forming the HLO 2 (develop new curricula for pilot training), which are relevant to the topic of this article and were fulfilled by project partners comprising Czech Technical University in Prague (CTU), Transport Research Institute Slovakia (VUD), University of Zilina (UNIZA), GNSS Centre of Excellence (GCE) and DSA.

It is a fact, that the integration of the SO3 concerning the pilot training in the utilization of EGNSS, their understanding of this matter and integrating GNSS among the other already standardized navigation systems is a key issue. It is not only important in the matter of safety, but also due to modern world evolution, where GNSS technologies have their safe place. On the basis of this evolution, the project worked towards an update in the pilot training in the matter of GNSS for all training modules for all pilot license types. Such progress leads to a guarantee of adequately trained pilots, with the ability to use all gained knowledge and experience with the newest navigation technologies to navigate the aircraft during flights or landings in any kind of severe weather.

Dissemination of information and increasing awareness about EGNSS between amateur and professional aviation community is a topic of the SO4. This was achieved by a set of information and awareness channels, targeting specific audience segments with particularly structured information and knowledge for an easy access by the meant audience. These include information and awareness channels such as internet channels (YouTube, Facebook, project website), physical inperson channels (workshops, trainings, events and multimedia education such as video series/e-learning), all the these mentioned channels were customized to meet specific needs of its users (language customization, training customization for different audiences, various format for an easy access etc.). All these activities were a necessary part of the CaBilAvi project, to continue to increase awareness and contribute to the dissemination of GNSS throughout Europe, which undoubtedly results in an increased standard of living in Europe.

Figure 1 shows a detailed activity flow prepared by GCE as the main project coordinator, for Work package (WP) 3 . The fulfilment of WP3 begun by structuring and defining requirements and information for the new training curricula during a workshop No. 1 and No. 2. This was followed by structure comparison and addition of new information for theory, simulator and practice (LAPL, PPL, PPL VFR FI and $(-H)$. Advisory procedures for VFR were prepared by CTU, all learning materials were prepared and coordinated by VUD, UNIZA and DSA.

\section{Work done}

\subsection{New curricula}

Based on CaBilAvi workshops No. 1 and No. 2 were generated and proposed changes to theoretical training syllabus for VFR pilots. The changes were outcomes of previous experience of experts from CTU, ATO's and pilot training organizations in the Czech Republic and Slovakia. To get an overview of current situation and its limitations, PPL training materials from ATO's were gathered and analyzed to later serve as a guiding material for a new syllabus with the proposed changes. Recommended time schedule for GNSS training for PPL(A) was prepared based on the outcomes of the analysis to assure sufficient time for pilots to understand and absorb new information. To meet the requirements of each license type (LAPL(A), LAPL(B), GPL, BPL and AsPL), customized time schedule and syllabus was prepared separately. As mentioned above, GNSS usage is increasing rapidly even without any proper training, which was concluded after a discussion with SPL and LAPL(S) license holders. As a result of this aforesaid, CTU has proposed certain recommendations to EASA legislation with regards to NPAS 2016-14 introducing new licenses for obtaining IR rating for VFR pilots. 


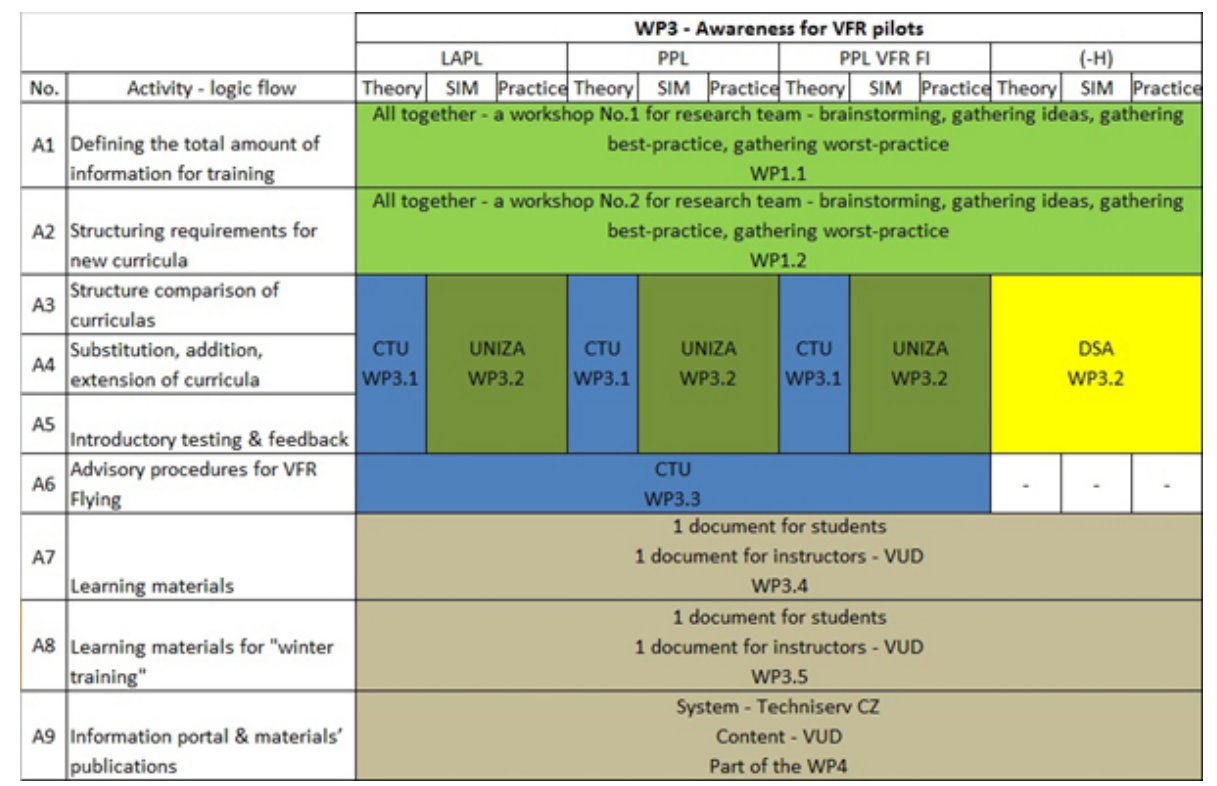

Figure 1. Activity logic flow regarding awareness dissemination for VFR pilots (work packages introduced by VUD, CTU, DSA and UNIZA)

The next step taken were recommendations for changes to practical training syllabus for VFR pilots. The syllabus was prepared by an expert team from UNIZA, who agreed that the main problem was not sufficiently comprehensive information about GNSS provided to VFR pilots. UNIZA prepared a new comprehensive learning material with respect to syllabus published by European Aviation Safety Agency (EASA) and based on the above mentioned theoretical training syllabus and especially upon the outcomes of practical and simulator testing performed at UNIZA Air Training and Educational Centre. Consequent to the simulator and practical testing with proposed changes and matters related to VFR GNSS utilization within GA community, which were discussed during a Winter training workshop in Slovakia by representatives of aviation sectors from the Czech Republic and Slovakia (CAA, ANS, GA, SAR, CAT), was finally as a result developed the practical training syllabus with all topics that are relevant and should be tested in order to meet the practical training skills for the applicable license types (LAPL, PPL, FI for VFR).[4][5]

Related to the new curricula shall be introduced the Advisory procedures to pilots without IR with reference to GNSS to maintain separation from obstacles, airspace structure and safe distance from other traffic. These procedures advice VFR pilots to use GNSS devices as a supplementary device in certain situations with obvious benefits (in CTR, TMA and Restricted airspace) to avoid loss of orientation, for safety of landing, for VFR GNSS approaches and for quick position altitude reference. For each of the stated situation was prepared a structured check-list, which could be adjusted by each operator with own advisory procedures. To prove that correct usage of GNSS with advisory procedures lowers the stress level of pilots, simulator testing flights were performed, while mon- itoring specific physiological parameters during flights with a GNSS device and during flight without any GNSS device. This testing has proved that the stress level has significantly decreased when using a GNSS device during flight.

\subsection{Learning materials}

To provide clear and comprehensive information about theoretical and practical skills regarding GNSS for VFR pilots, learning materials were prepared as a supportive material for students and instructors. The learning materials were introduced in a form of a book divided into two parts, Part A/B. Part A contains updated theoretical knowledge about GNSS, required for license types PPL(A), PPL(H) and LAPL, with information about GNSS operations, description of GNSS receivers for GA and other suggestions for a safe flight. Part B of the learning materials has a slightly different structure, whereas its structure reflects the proposed changes to the PPL(A), PPL(H) and LAPL theoretical knowledge syllabus with short information to each change or addition. The learning materials were published in printed version and in electronic version, available for download through OpenAire system via the Zenodo repository, with a generated DOI 10.5281/zenodo.1035189, it is also available on the CaBilAvi website.

These learning materials also served for the purpose of launching of an e-learning portal, which is an online learning application about GNSS for VFR pilots. The e-learning portal contains content based on the Part B of the leaning materials.

Together, the learning materials (Part A and Part B) and the e-learning portal make a comprehensive structured learning material, which shall be used by VFR pilots to adopt knew knowledge that is necessary for safe flying with GNSS.[6] 


\subsection{Learning materials for Winter training (WT)}

For the purpose of Winter training workshops, which took place in the Czech Republic, Slovakia and Spain, were created specific learning materials, introduced specifically at these events. These materials were prepared based on analysis of relevant legislation and materials with the GNSS topic already published during the project.

The very first learning material was Winter Training Brochure prepared by UNIZA for the purpose of Slovak Winter training with included materials for VFR/IFR flights and overall GNSS principles, basically all elemental knowledge, theoretical background, practical examples and advice for the use of GNSS devices for VFR and IFR flights (Part 1: Basic theoretical base and GNSS principles for VFR and IFR flights, Part 2: Principles of GNSS use for VFR flights, Part 3: Principles of GNSS use for IFR flights.). This Slovak brochure was later translated into English and used as a learning material at the Winter training in Spain and later at similar trainings across Europe.

Subsequent to the Winter training brochure were prepared materials for Winter training evets organized in the Czech Republic, these included presentations and training materials for pilots attending an obligatory Winter training event.

The Winter training brochure is available under ISBN 978-80-89923-02-1 with copyright to GSA, available in electronic version for download through the OpenAire system via the Zenodo repository, with a generated DOI 10.5281/zenodo.1035147. The brochure can also be accessed through the CaBilAvi website.

\subsection{Activities on aviation awareness and project dis- semination}

Activities described below were introduce during the $\mathrm{Ca}$ BilAvi project to support the project itself and specially to ensure, the project outcomes are distributed within a broad aviation community.

\subsubsection{Education video series}

One of the key activities for dissemination of the project outcomes was launching of a twenty-episode long (YouTube $\mathrm{CZ}$ /YouTube EN) educational video series on satellite navigation GNSS. As part of these series were weekly published videos pursuing the opportunities and benefits emerging the use of GNSS for general aviation pilots flying according to visual flight rules as well as for instrument flight rules flights. Besides the benefits, the videos are also mentioning threats and possible limits in the use of satellite navigation to prevent and eliminate critical situations in case pilots merely rely on use of such navigation. At the same time the channel has been linked to a Facebook profile with regularly published YouTube videos. The series is available in Czech language (original) and in in English language, making the videos and information more accessible for non-Czech speaking users.

To ensure the video series finds its right audience, it was made available through a YouTube profile of GCE and published on a Facebook profile of GCE, where the videos can also be freely accessed. Both of the accounts are in Czech and English modification to assure simple access to the content.

\subsubsection{National info packs}

Overall five different information packs explaining the benefits of EGNOS and the practical implications for relevant groups have been issued. Information packs target groups: airports and heliports, aircraft operators, pilots, rotorcraft and ATO's. These info packs are available in national languages of the countries involved in the project. These materials were distributed and used at Winter training events in Czech Republic. These information packs are available on the CaBilAvi website in various language modifications.[7]

\section{Conclusion}

This article shows a significant part of activities introduced during the CaBilAvi project endorsed in the 8th Framework Program of European Commission for Research and Development (HORIZON2020) under the EC number 641627, which were done by project partners from Czech Republic and Slovakia. To ensure safe airspace, it is necessary to constantly educate pilots and enable an access to current information. Therefore, the project made an important step for increasing safety in the European airspace by updating obviously obsolete syllabus for theoretical and practical VFR pilots training and by preparing learning materials directly submerged to pilots. To ensure the information is delivered to all concerned, the project has introduced a great concept of distributing the information through obligatory trainings and through an involvement of key aviation stakeholders such as ATO's or aircraft operators. Important part of the project was also in the supportive activities, which delivered the new gained knowledge through easily accessible sources to various audience interested in aviation.

\section{Acknowledgement}

This article has been produced thanks to the opportunity of Vladimir Socha and Lenka Hanakova. The information written above has arisen from authors involvement in the abovementioned project, therefore special thanks goes to authors mentor Tomas Dusa. The work in this part of the project was delivered by project partners from CTU, VUD, UNIZA and DSA, therefore thanks goes to all of the colleagues who participated and submitted such great work.

\section{References}

[1] Gnss - global navigation satellite system. URL http://www.czechspaceportal.cz/en/ section-3/gnss-systems/.

[2] Egnos. URL http://www. czechspaceportal. cz/en/egnos-1/. 
[3] European gnss drives general aviation. URL https://www.gsa.europa.eu/news/ european-gnss-drives-general-aviation.

[4] Vratislav; Michal BENES at al ELIAS. New curricula with gnss for vfr ppl (h) training: Capacity building for aviation stakeholders, inside and outside the eu - cabilavi. Technical report, 2017.

[5] Jakub HOSPODKA and Anna POLANECKA. New curricula with gnss for vfr training: Capacity building for aviation stakeholders, inside and outside the eu - cabilavi. Technical report, 2017.

[6] Roman TOPOLCANY Tomas BRACINIK JUN, Frantisek and Paulina HALJAKOVA. Recommendations for changes and additions to vfr flight training curricula: Capacity building for aviation stakeholders, inside and outside the eu - cabilavi. Technical report, 2017.

[7] Periodic technical report part b: Capacity building for aviation stakeholders, inside and outside the eu - cabilavi. Technical report, 2017. 